\title{
El lenguaje como herramienta al servicio del procés soberanista de Cataluña
}

\author{
Language as a tool in the service of the sovereigntist process of Catalonia
}

\author{
Alfedo CRESPO ALCÁZAR \\ ADESyD (Asociación de Diplomados Españoles en Seguridad y Defensa) - España \\ alfredocrespoalcazar1974@gmail.com
}

\begin{abstract}
Cómo citar este artículo: CRESPO ALCÁZAR, Alfredo (2016): «El lenguaje como herramienta al servicio del procés soberanista de Cataluña», Mediaciones Sociales, no 15, pp. 101-114. DOI: http://dx.doi.org/10.5209/MESO.54545
\end{abstract}

Recibido: 27 de junio de 2016.

Aceptado: 12 de septiembre de 2016.

\section{RESUMEN}

El proceso separatista puesto en marcha por los sucesivos gobiernos de Cataluña, en particular desde 2010, descansa en parte en un manejo deliberadamente ambiguo del lenguaje. Al respecto, el nacionalismo catalán evoca y repite conceptos polisémicos, tratando de dotar de legalidad y legitimidad a sus aspiraciones rupturistas. La ambigüedad desaparece cuando el objetivo es estigmatizar al adversario político, caracterizándolo de manera nítida como hostil y contrario a los intereses de Cataluña. El resultado es una dialéctica con la que el nacionalismo catalán busca la exoneración de cualquier responsabilidad.

PALABRAS CLAVE: derecho a decidir, independencia, nacionalismo catalán, referéndum, soberanía.

\section{ABSTRACT}

The separatist process carried out by the successive governments of Catalonia, particularly since 2010, rests in part on a deliberately ambiguous management of language. In this respect, Catalan nationalism evokes and repeats polysemic concepts, trying to provide legality and legitimacy to its breaking aspirations. The ambiguity disappears when the objective is to stigmatize the political adversary, characterizing it in a clear way as hostile and contrary to the interests of Catalonia. The result is a dialectic with which Catalan nationalism seeks the exoneration of any responsibility.

KEYWORDS: right to decide, independence, Catalan nationalism, referendum, sovereignty. 
Sumario: 1. Introducción. 2. Los prolegómenos del procés: la reforma del Estatuto de Autonomía (2003-2010). 3. Nuevos conceptos, nuevas exigencias (2010-2015). 4. El lenguaje como herramienta al servicio de la legitimación de un proyecto político particular. 5. Conclusiones. Bibliografía.

\section{INTRODUCCIÓN}

La política en Cataluña no discurre por los mismos derroteros que en otras comunidades autónomas españolas. El eje derecha-izquierda convive con el de nacionalismo-no nacionalismo. La coexistencia de ambos ejes, si bien ha sido una constante desde las elecciones autonómicas de 1980, se intensificó durante la etapa 2003-2010 por influencia de los dos gobiernos tripartitos, una alianza gubernamental de partidos políticos no independentistas (PSC e ICV) ${ }^{1}$ e independentistas (ERC) $)^{2}$. Esta novedosa fórmula de gobierno, en cuanto que durante los 23 años anteriores Cataluña había conocido únicamente la hegemonía gubernamental de $\mathrm{ClU}^{3}$ (no siempre con mayorías absolutas), unido a la prioridad que el Tripartito concedió a la reforma del Estatuto de Autonomía de 1979 (Estatuto de Sau), convirtió a esta comunidad autónoma en uno de los principales centros de interés de la vida política española.

Este fenómeno se acentuó a partir de 2010, cuando CIU regresó al gobierno de Cataluña, iniciando un proceso soberanista (el procés) en el que no ha escatimado medios. Esto le ha permitido denunciar una situación que califica de injusta (la relativa a su estatus constitucional dentro de España), así como compararse con otros escenarios que, según considera, presentan similares características a las de Cataluña. Dentro de estos últimos, Escocia y su referendo in vs out de 2014 ha resultado el más significativo, como antes lo habían sido los de los países bálticos (Vidal-Folch, 2014: 87). La claridad con que el Scottish National Party ha defendido históricamente la independencia de Escocia contrasta, sin embargo, con la ambigüedad a la que ha recurrido $\mathrm{CIU}$.

Para llevar a cabo su proyecto político, el soberanismo ha creado un sofisticado entramado institucional (por ejemplo, el Consejo Asesor para la Transición Nacional), ha potenciado las actividades de entidades que responden a sus objetivos independentistas (como el Institut Nova Historia o el Centro de Estudios Contemporáneos de

\footnotetext{
1 PSC: Partit dels Socialistes de Catalunya / Partido de los Socialistas de Cataluña.

ICV: Iniciativa per Catalunya Verds / Iniciativa por Cataluña-Los Verdes.

2 ERC: Esquerra Republicana de Catalunya / Izquierda Republicana de Cataluña.

3 CIU: Convergencia i Unió / Convergencia y Unión.
} 
la Generalitat) y ha subvencionado eventos como el simposio "España contra Cataluña" celebrado en 2013.

En este proceso, el lenguaje ha sido uno de los principales instrumentos a través de los cuales el nacionalismo catalán ha canalizado su apuesta rupturista. Durante la última década, ha construido un relato hegemónico articulado, como veremos, en torno al eje "nosotros vs. ellos". Para el nacionalismo, Cataluña ha hecho todo lo posible por encontrar su "encaje en España"; pero, como el Estado español no da respuesta a las aspiraciones catalanas, la única salida que queda es la independencia. Sobre esa base, una concepción excluyente de la identidad catalana, el uso ambiguo del concepto de independencia o la construcción de España como enemigo son algunos de los componentes que conforman el discurso nacionalista puesto al servicio del procés.

A lo largo de las siguientes páginas, analizaremos cómo el nacionalismo catalán, en su tránsito desde posiciones autonomistas y pactistas hacia posturas soberanistas, ha empleado el lenguaje para articular sus ideas y objetivos favorables a la independencia, en la línea de otros estudios que vinculan discurso y nacionalismo (por ejemplo, Beobide, 2003; Cabrera, 1992; Martínez, 2011; Rubio, 2015; Zambelli, 2015).

Para nuestra exposición, seguiremos un orden cronológico, estableciendo dos etapas. La primera, comprendida entre 2003-2010 y que corresponde a los dos gobiernos tripartitos, estuvo protagonizada por la reforma del Estatuto de Autonomía de 1979. La segunda se inicia con el retorno de CIU al gobierno tras las elecciones autonómicas de 2010 y se extiende hasta la actualidad; se caracteriza por una mayor radicalidad de los planteamientos nacionalistas, sobre todo, a partir de 2012. En cada una de ellas han ido irrumpiendo nuevos actores políticos que, a través de sus acciones (en forma de reivindicaciones) e interacciones han añadido dosis de complejidad al escenario catalán.

\section{Los prolegómenos del procés: la Reforma del Estatuto de Autonomía (2003- 2010)}

La reforma del Estatut fue el tema que monopolizó las dos legislaturas del Tripartito (2003-2006, 2006-2010). Esta reforma estatutaria se llevó a cabo a través de un largo proceso que generó elevadas dosis de conflicto en las relaciones entre el gobierno de Cataluña primero con el ejecutivo central presidido por José María Aznar $\left(\mathrm{PP}^{4}\right)$ y posteriormente por José Luis Rodríguez Zapatero $\left(\mathrm{PSOE}^{5}\right)$.

\footnotetext{
4 PP: Partido Popular.

5 PSOE: Partido Socialista Obrero Español.
} 
Cronológicamente, el texto de reforma fue aprobado primero en el Parlamento de Cataluña el 30 de septiembre de 2005, con la única oposición del PPC ${ }^{6}$, que consideraba innecesario modificar el Estatuto. Tampoco para la sociedad catalana era una prioridad. La reforma parecía más un deseo de la clase política catalana, en particular del PSC, ICV y ERC, para diferenciarse del pujolismo. En este sentido, cabe recordar que Jordi Pujol, líder histórico del nacionalismo catalán y presidente de la Generalitat entre 1980 y 2003, se había opuesto a la reforma del Estatut durante su última legislatura (1999-2003).

Durante dicha legislatura, PSC, ERC e ICV dispusieron del margen de tiempo suficiente para difundir un discurso político que, en lo esencial, acusaba al PP de aumentar el centralismo, recalcaba la importancia de las políticas sociales y exigía la reforma del Estatuto de 1979.

CIU, en un escenario interno marcado por la designación de Artur Mas como sucesor de Pujol, acabó sumándose a las críticas al gobierno del PP, valorando negativamente una colaboración iniciada en 1996 con el Pacto del Majestic (por el que Jordi Pujol prestó su apoyo a la investidura de José María Aznar) y continuada en la Generalitat de Cataluña entre 1999-2003, al no disponer los convergentes de mayoría absoluta. Así, CIU se fue aproximando a las tesis de Pascual Maragall (PSC), presidente de la Generalitat entre 2003 y 2006, acerca de la recentralización que, bajo su punto de vista, estaba realizando Aznar al frente del gobierno central. De hecho, el ejemplo más claro del paulatino viraje convergente lo encontramos en su posición hacia el Estatut: de oponerse a dicha reforma a defenderla como elemento central de su programa electoral en las elecciones autonómicas de 2003.

Con todo ello, en Cataluña se fue gestando una suerte de frente "reformista" integrado por PSC, ERC, ICV y CIU, que contaba con el apoyo de Rodríguez Zapatero, tanto cuando éste se encontraba en la oposición (2000-2004) como cuando se convirtió en presidente del gobierno de España (2004). Maragall y Rodríguez Zapatero compartían conceptos como los de "España nación de naciones" o "España plural".

El Estatut fue aprobado el 30 de marzo de 2006 por las Cortes Generales, con el voto en contra del PP y la abstención de ERC, que consideraba el contenido final insuficiente. Tras ello, el PP puso un recurso de inconstitucionalidad a la totalidad, ya que, en opinión de su presidente, Mariano Rajoy, el nuevo texto "liquidaba unilateralmente el modelo de Estado" (Agencias, 2006).

En Cataluña, la reforma se sometió a un referendo que tuvo lugar el 18 de junio de 2006. Venció el Sí al Estatut con el $74 \%$ de apoyo, aunque la participación no alcanzó el 50\%. En el referendo, ERC -socio de los socialistas en el gobierno catalán- pidió el

\footnotetext{
6 PPC: Partido Popular de Cataluña.
} 
voto en contra. Esta decisión provocó su expulsión del gobierno de Maragall, quien al quedarse en minoría hubo de convocar elecciones en 2006. En ellas, su lugar como candidato del PSC lo ocupó José Montilla.

CIU resultó ganadora en la contienda electoral, como en 2003, aunque de nuevo no obtuvo el número de escaños suficientes para gobernar. PSC, ICV y ERC sí que sumaron los escaños necesarios para constituir un nuevo gobierno tripartito, presidido en esta ocasión por Montilla. Durante la campaña electoral, CIU recurrió a un lenguaje contundente para referirse a sus adversarios políticos, sobre todo, hacia el $\mathrm{PP}$, al que acusaba de ir contra Cataluña para lograr votos en otras partes de España. Mas, candidato convergente, incluso acudió al notario para ratificar que, fueran cuales fueran los resultados de esos comicios, nunca pactaría con el PP.

Esta segunda legislatura del Tripartito (2006-2010) estuvo influida en todo momento por el retraso del Tribunal Constitucional a la hora de emitir la sentencia sobre el recurso que habían puesto al Estatut, entre otros, el PP y el Defensor del Pueblo (Fernando Múgica). Conforme se demoró la sentencia, Cataluña se convirtió en escenario de manifestaciones independentistas (como por ejemplo la desarrollada en Barcelona a finales de junio de 2009 bajo el lema Som una nació, que exigía una declaración unilateral de independencia), referendos ilegales (como el celebrado en Arenys de Mar en septiembre de 2009), desafíos permanentes al entramado de derechos y libertades consagrados en la Constitución y frecuentes descalificaciones al Tribunal Constitucional, como la proferida por el entonces aún miembro del PSC Ernest Maragall:

«¿ंPor qué concedemos tanta significación a la famosa y temida sentencia del Tribunal Constitucional español? Digámoslo claro y en voz alta: el pacto Cataluña-España está cerrado y rubricado. Aún más, está confirmado por la única voz indiscutible e incontestable: la voz de los ciudadanos refrendando el acuerdo entre los parlamentos catalán y español. ¿Qué puede añadir "la interpretación" que hagan, por larga y enrevesada que sea, este grupo de ciudadanos tan sabios?» (Maragall, 2009).

La sentencia del Tribunal Constitucional se produjo en junio de 2010. No dejó satisfechos a los valedores del Estatut en Cataluña y sí en mayor medida al gobierno de España y al PP. El fallo declaró inconstitucionales 14 artículos y realizó restricciones interpretativas sobre otros 30 , por lo cual no se podía hablar de su plena constitucionalidad (Wert, 2010).

ERC no aceptó la sentencia. El presidente Montilla, por su parte, convocó una manifestación bajo el lema Som una nació. Nosaltres decidim (Somos una nación. Nosotros decidimos), recogiendo el sentir de amplios sectores del PSC, que consideraron la decisión del Constitucional una provocación a Cataluña, como por ejemplo Jordi Mercader (PSC): 
«Cataluña hace una propuesta a España. España se espanta y retoca esa propuesta. Cataluña acepta, la somete a referéndum, la ciudadanía dice que sí, pero España nuevamente vuelve a retocar la propuesta. La afrenta a Cataluña es o fue, pues, brutal» (Mercader, cit. en San Agustín, 2014: 169).

\section{NuEVOS CONCEPTOS, NUEVAS EXIGENCIAS (2010-2015)}

En 2010, se celebraron en Cataluña nuevas elecciones autonómicas. Pese al malestar señalado entre los socialistas catalanes por la sentencia del Tribunal Constitucional sobre el Estatut, de cara a los comicios se apreció un viraje del PSC hacia posturas "más españolistas", con las que pretendía contrarrestar el avance soberanista de CIU y desmarcarse de ERC, su socio en el Tripartito.

CIU ganó las elecciones con un programa en el que el pacto fiscal (esto es, un nuevo modelo de financiación autonómica para Cataluña) ocupó el lugar más destacado. A pesar de no lograr la mayoría absoluta, esta vez sí pudo gobernar. Durante la campaña electoral moderó, aunque no eliminó, sus aspiraciones soberanistas (Canal, 2010). Una vez en el gobierno, estas aspiraciones afloraron envueltas en elevadas dosis de victimismo.

Artur Mas, nuevo presidente de la Generalitat, privilegió un discurso más pragmático que "nacional" que le servía para criticar la herencia recibida del gobierno tripartito (en forma de una economía y unas relaciones con el gobierno central deterioradas). En sus intervenciones previas a las elecciones de 2010, fue recurrente que subrayara la importancia de mejorar las infraestructuras, de internacionalizar el tejido empresarial, de dotar de agilidad a la administración pública (sin olvidar la reducción de su tamaño), de fomentar valores como el esfuerzo y el talento. Pero también avisó de la actitud que iba a mostrar Cataluña en los próximos años con respecto a sus relaciones con España:

«La España de hoy no es atractiva, no nos protege, sólo nos pide, no nos acepta tal como somos, no respeta los votos del pueblo catalán cuando pide un cierto nivel de autogobierno. Por eso, Cataluña ha establecido una hoja de ruta: no a la vía de los últimos 30 años porque nos han cerrado la puerta los grandes poderes del Estado, haremos una transición hacia otro rumbo. Cataluña no puede renunciar a su progreso como país, como nación y como sociedad» (Mas, 2010).

La parte final de la argumentación de Mas no difería mucho de la lectura que en idéntico escenario (la Tribuna Nueva Economía) había hecho unos meses antes JosepLluis Carod-Rovira (ERC), vicepresidente de la Generalitat durante el segundo gobierno tripartito. Carod-Rovira rechazaba el federalismo (porque lo entendía como una forma de mantener la unidad de España), exigía que Cataluña fuera reconocida como sujeto político y económico, para concluir finalmente que "los catalanes quie- 
ren construir Estado y si este Estado no es España, tendrán que buscar otras fórmulas. A Madrid no siempre llega con nitidez lo que sucede en Cataluña donde se está produciendo una construcción" (Carod-Rovira, 2010).

Durante los primeros compases del primer gobierno de Mas, en parte motivado por los pactos con el PPC para sacar adelante los presupuestos de la Generalitat de 2012 (Pacto del Neri), CIU priorizó los aspectos de política práctica sobre los identitarios, lo cual no implicaba que estos hubieran quedado olvidados, como se demostró a partir de la segunda mitad de 2012, con la exigencia de un pacto fiscal al gobierno central. Igualmente, en la manifestación del 11 de septiembre de 2012 (realizada en clave soberanista y organizada por la Asamblea Nacional de Cataluña, organismo creado ese mismo año para defender y promover la independencia), CIU tuvo un papel protagonista, participando los principales referentes del partido (Jordi Pujol, Oriol Pujol, Josep Antoni Durán i Lleida...), aunque no Artur Mas.

Esta acentuación del perfil soberanista por parte de CIU hizo que la colaboración inicial con el PPC retrocediera hasta desaparecer. En palabras de Alicia SánchezCamacho, presidenta del PP catalán:

«El catalanismo moderado de CIU ha desaparecido y ha sido sustituido por la separación de Cataluña del resto de España mediante la búsqueda del Estado propio y de la independencia. Esa es la línea ideológica de CIU. Responde a la ideología de las nuevas generaciones de $\mathrm{CDC}^{7}$ lideradas por Oriol Pujol Ferrusola» (Sánchez Camacho, cit. en Cañizares, 2012).

A partir de ese momento, la independencia se convirtió en el objetivo principal para el gobierno de Cataluña, refiriéndose a ella a través de diferentes subterfugios léxicos como "derecho a decidir", "interdependencia" o "estructuras de Estado". De hecho, en el programa electoral presentado por CIU para las elecciones autonómicas de 2012, la independencia no apareció en ningún lugar, optando por unir argumentos identitarios y económicos con la finalidad de presentarla de una forma que resultara atractiva para el votante.

La apuesta de CIU por la independencia encontró apoyo en otras formaciones que históricamente habían mostrado su total compromiso con la independencia, como ERC o Solidaridad Catalana por la Independencia.

El nuevo líder de ERC, Oriol Junqueras, se unió a la tesis defendida por CIU del "expolio fiscal" a Cataluña -expresión más elaborada que el habitual "España nos roba" del discurso nacionalista, pero con idéntico contenido y significado-, añadiendo que una victoria de ERC equivaldría a la creación automática de un Estado catalán.

\footnotetext{
7 CDC: Convergència Democràtica de Catalunya / Convergencia Democrática de Cataluña, socio principal de la coalición CIU, la cual se disolvió en 2015.
} 
En cuanto a la postura de Solidaridad Catalana por la Independencia, el discurso de su candidato, Alfons López Tena, era más radical que el de CIU y ERC. Acusaba a ambas formaciones de no querer la independencia, sino el autonomismo:

"CIU quiere mantenerse en España y sólo habla de interdependencia o de ser como Massachussets o Baviera, sin tener una hoja de ruta clara. Y ERC tiene un problema de credibilidad: habla de independencia desde 1992 pero en la práctica apoyan el pacto fiscal, que es pactar con España» (López Tena, cit. en Pi, 2012).

Solidaridad Catalana por la Independencia rechazaba que se produjera fractura social alguna en Cataluña con el proceso soberanista y centraba sus recursos dialécticos en acusar a España de robar a Cataluña y de ser la responsable de sus principales males, que desaparecerían con la independencia (López Tena, 2009; Strubell, 2009).

\section{EL LENGUAJE COMO HERRAMIENTA AL SERVICIO DE LA LEGITIMACIÓN DE UN PROYECTO POLÍ- TICO PARTICULAR}

A pesar de que CIU no logró "la mayoría excepcional" que reclamaba en los comicios autonómicos de 2012, ello no supuso que rebajara o alterase sus reivindicaciones soberanistas; por el contrario, las incrementó desde entonces, con la colaboración de otras fuerzas políticas, en particular ERC y las CUP ${ }^{8}$. La consulta del 9 de noviembre de 2014, la Declaración de Soberanía aprobada por el Parlamento catalán en enero de 2013 o el Pacto Nacional por el Derecho a Decidir constituido a mediados de este mismo año corroboran dicha afirmación. Complementariamente, a partir de 2012 el soberanismo multiplicó el empleo de la simbología, principalmente a través de las manifestaciones de cada 11 de septiembre (coincidiendo con la celebración de la Diada, fiesta de Cataluña), interpretadas como ejemplo de su capacidad de resistencia.

En ese proceso, ha sido habitual el recurso al lenguaje como una herramienta más al servicio de la independencia. El nacionalismo catalán ha articulado diferentes estrategias discursivas para construir simbólicamente la nación catalana, legitimar su apuesta soberanista y, a la vez, deslegitimar el marco de convivencia emanado de la Constitución de 1978. Ha elaborado para ello un relato cuyos componentes esenciales se articulan en torno al eje dialéctico "nosotros vs. ellos".

Bajo la perspectiva del nacionalismo, el Estado español desprecia las aspiraciones, instituciones y cultura catalanas. España habría fallado a Cataluña: ésta siempre estuvo al servicio de la modernización democrática y económica del conjunto del país (Pujol, 2001: 18-21), pero a cambio sólo recibió incomprensión (Bel, 2013). Expresio-

\footnotetext{
8 CUP: Candidatura d'Unitat Popular / Candidatura de Unidad Popular.
} 
nes como "España nos roba" muestran dónde se sitúa el nacionalismo en su relación con España (como víctima) y donde ubica la responsabilidad de los problemas de la sociedad catalana (en España).

La idea de que Cataluña está siendo despreciada por las instituciones españolas encuentra para el nacionalismo su ejemplo paradigmático en el trato recibido durante el proceso de elaboración y aprobación del nuevo Estatut (Ridao, 2014: 19). La modificación del texto en las Cortes españolas y el rechazo de algunos artículos por parte del Tribunal Constitucional demostraron, desde el punto de vista nacionalista, que el encaje de Cataluña en España era imposible (López, 2014), por razones siempre imputadas a la segunda (Laínz, 2014).

De hecho, la consideración de que la vía federal es una vía muerta se ha convertido en uno de los ejes del discurso nacionalista. Así, para Jaume López:

«Si el independentismo tiene fuerza hoy en Cataluña es sobre todo por el fruto de una constatación: ni protestas, ni propuestas (como la del Estatut) tienen ninguna opción real de prosperar en esta España. Tal como muestran todas las encuestas, la mayoría de los catalanes ha tenido como primera preferencia hasta muy recientemente el federalismo (...) ¿Se reformará el Estado español? Hoy la lógica (no la ideología o el deseo) nos lleva a pensar que no» (López, 2014: 241 y 243).

Joan Ridao se expresa en similares términos:

«Sin duda, la estocada final fue la Sentencia del Tribunal Constitucional sobre el Estatuto de Cataluña de 28 de junio de 2010. Esa resolución del Alto Tribunal español supuso un correctivo sin paliativos que acabó con los argumentos de quienes todavía creían en la posibilidad de un ensamblaje federal en el marco de la ambigüedad heredada de la Transición. La respuesta política y cívica ante la sentencia fue contundente» (Ridao, 2014: 26).

El nacionalismo catalán ha reiterado, por tanto, el argumento de que ha buscado "el encaje en España" pero al no conseguirlo, por causas imputables sólo a España, se ha visto obligado a optar por la independencia (Bel, 2013: 123). Para el independentismo, si cada día existe un mayor número de castellanoparlantes que se adhieren a la emancipación nacional, es por la cerrazón del poder central (Bosch, 2014: 35).

Desde ese punto de vista, la "consulta" y el "derecho a decidir" constituían la reacción lógica a la desafección y al desencanto de Cataluña hacia España. Así, desde noviembre de 2012, en el vocabulario del nacionalismo catalán, el protagonismo principal ha correspondido a la expresión "derecho a decidir", que encierra una defensa del derecho de autodeterminación. Ambos, aunque no lo sean, se emplean como sinónimos (Ridao, 2014: 11), si bien como sostenía Agustí Colomines (cit. en Agenjo, 2016: 242), con un nivel de claridad nada habitual, "eso del derecho a decidir es una chorrada que nos inventamos para no decir lo que es, que es derecho de au- 
todeterminación, y punto pelota". Encontramos aquí una de las características fundamentales del discurso nacionalista: el uso ambiguo de la polisema como herramienta de persuasión. Consciente de que el empleo del término "independencia" podría resultar conflictivo, el nacionalismo ha evitado deliberadamente su uso, optando por expresiones como "Cataluña nuevo Estado de la Unión Europea", "estructuras de Estado" (catalán), "desafección de Cataluña hacia España", "desconexión de Cataluña" (con respecto a España) y, más recientemente, "derecho a decidir", una suerte de visión edulcorada del derecho de autodeterminación, que ha contado con el apoyo de sectores académicos, periodísticos e intelectuales españoles que han entendido legítima dicha reivindicación (Cruz, 2014; Boadella, 2013: 57). Lo mismo cabe afirmar del uso ambiguo del término "consulta" para referirse al referendo del 9 de noviembre de 2014.

Como parte de la construcción simbólica de la nacionalidad catalana, encontramos también la sustitución reiterada del nombre de "España" por la expresión "el Estado español", que lleva implícito el no reconocimiento de España como nación:

«Desde los orígenes el nacionalismo catalán ha definido a Cataluña como una nación y España como un Estado: lo natural frente a lo artificial. De ahí, por ejemplo, la insistencia, en los últimos tiempos, en muchos sectores en evitar nombrar a España y, en su lugar, referirse al Estado español. El nacionalismo, por definición, aspira a hacer coincidir hoy, mañana o algún día, la nación con el Estado, esto es, a convertirse en Estado. Por lo tanto, no deben sorprendernos las evoluciones hacia posiciones soberanistas o independentistas. No existen límites. De hecho, esta circunstancia, precisamente, les permite estar en permanente movilización y regeneración. La reclamación es siempre una apuesta ganadora: si se consigue, estamos ante un triunfo merecido; si no se consigue estamos ante una nueva injusticia, que alimenta evidentemente el (re)sentimiento nacionalista» (Canal, 2011: 66).

El procés soberanista ha fomentado así un relato que concibe a Cataluña y a España como entidades antagónicas. Cabe señalar, en todo caso, que el origen de este proceso de desconexión simbólica con el Estado español no se encuentra, en realidad, en 2010, sino que tiene una larga trayectoria. Su inicio cabe situarlo en 1980, con la llegada al gobierno de la Generalitat de Jordi Pujol (De Carreras, 2014). Es el fenómeno que Jordi Canal (2008) define como "renacionalización" de la sociedad catalana, en función del cual, una parte importante de la misma ha terminado por aceptar tres afirmaciones: que Madrid roba a Cataluña; que la política catalana es diferente; $y$, finalmente, que Cataluña es más moderna y más europea que España.

Las tres ideas apuntadas por el profesor Canal se han intensificado en los últimos tiempos, en paralelo a un desarrollo del victimismo. Para ello, el lenguaje ha resultado una herramienta fundamental, ya que ha permitido al nacionalismo catalán consolidar la idea de que está siendo "perseguido" y "amenazado" por las instituciones es- 
pañolas, por ejemplo, cuando estas han reaccionado judicialmente contra quienes participaron en la mencionada consulta del 9-N de 2014.

\section{CONCLUSIONES}

El nacionalismo catalán ha intensificado -como parte del procés soberanista iniciado en 2012- un relato que apuesta por presentar a España y a Cataluña como dos realidades irreconciliables. La negativa del gobierno central a satisfacer las aspiraciones de autogobierno de Cataluña ha servido al soberanismo para otorgar legitimidad a su proyecto independentista, aunque para ello se haya tenido que apoyar, como hemos señalado, en uso ambiguo del lenguaje.

La influencia del nacionalismo sobre los medios de comunicación y sobre un buen número de entidades público-privadas ha facilitado que su discurso se convierta en hegemónico y, a la vez, excluyente. La imagen de una Cataluña homogénea y única que se articula en dicho discurso contrasta con la heterogeneidad que caracteriza a esta comunidad autónoma. El nacionalismo emplea los vocablos “catalán” y "Cataluña" para referirse a quienes sintonizan con sus aspiraciones, omitiendo de este modo la existencia de sectores con posiciones antagónicas. Quien no ha compartido el discurso nacionalista ha optado en la mayoría de las ocasiones por el silencio como respuesta, debido a que cualquier postura disidente podría acarrear rechazo social. En consecuencia, numerosos ciudadanos no se atreven a exponer en público lo que sí hacen en privado (De Carreras, 2014). Juan Carlos Girauta, actual portavoz de Ciudadanos en el Congreso de los Diputados, explicaba este fenómeno en los siguientes términos:

«Cuando Artur Mas liquidó anticipadamente su primera legislatura y aireó su nuevo objetivo, la consecución de un "Estado propio", [el periodista Arcadi] Espada consideró que era un buen momento para que una serie de personalidades catalanas a las que se presumía contrarias a la independencia se pronunciaran. Treinta y siete fueron los interrogados (...). La respuesta inmediata de dieciséis de los personajes fue... que no responderían a las preguntas. Otros dos ni siquiera acusaron recibo. Otros se mostraron bien dispuestos, pero adujeron no saber qué contestar. Al fin, sólo dos personas pronunciaron claramente el "sí" (que en una consulta sobre la secesión correspondería al "no")» (Girauta, 2013: 16-17).

Al otorgar la condición de catalanes únicamente a aquellos que comulgan con sus exigencias y expectativas, el nacionalismo no sólo ha invisibilizado a la parte de la sociedad catalana no nacionalista. Ha sentado, además, las bases para la división social, como explica por ejemplo Ricardo Fernández Aguilá (2014: 30): “¿Qué se había hecho de los que nunca habían dicho que quisieran un Estado independiente para 
Cataluña? ¿De los que no se habían cansado de España, o al menos no mucho? ¿De los que sentían afecto por mucha gente más allá de casa nostra?".

La fractura en la sociedad catalana supone, hasta la fecha, el logro principal que ha producido esta dinámica, lo que se traduce en una estigmatización de España, de sus leyes y de sus instituciones. Desobedecer a las instituciones españolas, el nacionalismo catalán lo concibe como parte de un mandato democrático, al contemplar exclusivamente la voluntad de aquellos que vía electoral le otorgan su confianza.

\section{BIBLIOGRAFÍA}

AGENCIAS (2006): "El PP presenta su recurso de inconstitucionalidad contra la reforma del Estatuto Catalán", El País, 31 de julio de 2006. Disponible en https://goo.gl/BmLLim. Consultado el 16 de junio de 2016.

AGENJO, D. (2016): iSOS! Secuestrados por el nacionalismo. Madrid: La Esfera de los Libros.

BEL, G. (2013): Anatomía de un desencuentro. La Cataluña que es y la España que no pudo ser. Barcelona: Destino.

BEOBIDE, I. M. (2003): Prensa y nacionalismo vasco. El discurso de legitimación nacionalista. Bilbao: Universidad de Deusto.

BOADELLA, A. (2013): Diarios de un francotirador. Mis desayunos con ella. Barcelona: Espasa.

BOSCH, A. (2014): Como amigos. La independencia de Cataluña interesa a los españoles. Barcelona: Galaxia Gutemberg.

CABRERA, J. (1992): La nación como discurso. La estructura del sistema ideológico nacionalista: el caso gallego. Madrid: CIS / Siglo XXI.

CANAL, J. (2008): "Filosofías del nacionalismo", El Imparcial, 30 de junio de 2008. Disponible en https://goo.gl/X5mZTw. Consultado el 16 de junio de 2016.

CANAL, J. (2010): “¿Y cómo es él? Desenmascarando a Artur Mas", El Imparcial, 19 de abril de 2010. Disponible en https://goo.gl/2RtLpv. Consultado el 16 de junio de 2016.

CANAL, J. (2011): "El Estado autonómico: reflexiones históricas sobre Cataluña y el nacionalismo catalán", Cuadernos de Pensamiento Político, no 32, pp. 59-71.

CAÑIZARES, M. J. (2012): "Alicia Sánchez-Camacho: «Artur Mas ya ha dado los primeros pasos hacia la ruptura con España»", $A B C, 2$ de abril de 2012. Disponible en https://goo.gl/Yw345r. Consultado el 16 de junio de 2016.

CAROD-ROVIRA, J. L. (2010): "Intervención en el Foro de la Nueva Economía", 10 de febrero de 2010. Disponible en https://goo.gl/r3lpTa. Consultado el 16 de junio de 2016.

CRUZ, M. (2014): Una comunidad ensimismada. Madrid: Catarata. 
DE CARRERAS, F. (2014): Paciencia e Independencia. La agenda oculta del nacionalismo catalán. Barcelona: Ariel.

FERNÁNDEZ AGUILÁ, R. (2014): Un Fernandes entre banderas. Cuando ser catalán y español es una apuesta posible. Barcelona: Península.

GIRAUTA, J. C. (2013): Votaré NO a la secesión de Cataluña. Barcelona: Ediciones B.

LAÍNZ, J. (2014): España contra Cataluña. Historia de un fraude. Madrid: Encuentro.

LÓPEZ, J. (2014): La independencia de Cataluña explicada a mis amigos españoles. Treinta tópicos sobre la independencia catalana. Barcelona: RBA.

LÓPEZ TENA, A. (2009): Cataluña bajo España. La opresión nacional en democracia. Barcelona: Deria.

MARAGALL, E. (2009): “Construir Cataluña”, El País, 25 de agosto de 2009.

MARTÍNEZ, R. (2011): El lenguaje en la política vasca: tres corrientes, tres lenguajes (19962004). Bilbao: Universidad del País Vasco. Tesis doctoral.

MAS, A. (2010): "Intervención en el Foro de la Nueva Economía", 21 de septiembre de 2010. Disponible en https://goo.gl/bh84zD. Consultado el 16 de junio de 2016.

MONTILLA, J. (2010): "Intervención en el Foro de la Nueva Economía”, 13 de octubre de 2010. Disponible en https://goo.gl/kDHWqF. Consultado el 16 de junio de 2016.

PI, J. (2012): “Alfons López Tena: "España es un país que quiero, pero no es el mío»", La Vanguardia, 16 de noviembre de 2012. Disponible en https://goo.gl/awFbz. Consultado el 16 de junio de 2016.

PUJOL, J. (2001): “Identidad, cohesión y cambio social”, Hermes, no 2, pp. 18-21.

RIDAO, J. (2014): El derecho a decidir. Una salida para Cataluña y para España. Barcelona: RBA.

RUBIO, J. A. (2015): Decir nación. Idearios y retóricas de los nacionalismos vasco y catalán (1980-2004). Cáceres. Universidad de Extremadura.

SAN AGUSTÍN, A. (2014): Cuando se jodió lo nuestro: Cataluña-España crónica de un desencuentro. Barcelona: Península.

STRUBELL, T (2009): Hasta aquí hemos llegado. Claves para entender el hartazgo de Cataluña con España. San Sebastián: Ttarttalo.

VIDAL-FOLCH, X. (2013): ¿Cataluña independiente? Madrid: Catarata.

VIDAL-FOLCH, X. (2014): Cataluña ante España. Madrid: Catarata.

WERT, J. I. (2010): “Estatuto, lógica y sintaxis”, La Rioja, 4 de julio de 2010.

ZAMBELLI, S. (2015): El discurso nacionalista en Cataluña. Un análisis comparado de los marcos de significación del tardo-pujolismo (2000-2003) al proceso soberanista (2010-2014). Madrid: UNED. Tesis doctoral. 
Alfredo CRESPo ALCÁZAR es doctor por la Universidad Rey Juan Carlos de Madrid. Licenciado en Ciencias Políticas (UNED) y en Ciencias de la Información (UCM). Vicepresidente segundo de ADESyD (Asociación de Diplomados Españoles en Seguridad y Defensa) e investigador agregado del Instituto de Estudios Riojanos. Autor de Cameron. Tras la senda de Churchill y Thatcher (Logroño: Siníndice, 2011). Colaborador de diferentes medios de comunicación y revistas académicas, ha participado en numerosos proyectos de investigación (Instituto de Estudios Riojanos, Universidad Rey Juan Carlos de Madrid, Escola Galega de Administración Pública) e impartido comunicaciones y ponencias en una veintena de congresos nacionales e internacionales. Correo electrónico: alfredocrespoalcazar1974@gmail.com. 